\title{
DIVERSIDADES CUESTIONADORAS: LA CAMPAÑA INTERNACIONAL «STOP TRANS PATOLOGIZACIÓN 2012»
}

\author{
Aitzole ARANETA ZinkUneGi \\ San Sebastián
}

Recibido/12/03/2012

Aceptado/24/05/2012

\section{Resumen}

En los últimos años ha saltado a la agenda pública el concepto de «despatologización trans», gracias al surgimiento de un movimiento internacional que aboga por deconstruir arraigados estereotipos de género, que son los que llevan a este colectivo a ser tildado de «enfermo», en medio de un imbricado sistema de imperativos legales, médicos y económicos. En este artículo se realiza un análisis general, sobre las causas que han llevado, bajo la inminente publicación de las nuevas versiones de los manuales de enfermedad internacionales DSM-5 y CIE-11, a la visibilización de estos hechos. Deslavazando diferencias sustanciales en un conjunto de países que denominaremos «del norte» y «del sur», para pasar después al análisis de los últimos acontecimientos acaecidos, considerándolos pistas de lo que puede suceder en un futuro inminente a este respecto.

Palabras clave: APA, CIE, DSM, OMS patologización, trans, teoría queer.

\begin{abstract}
In recent years the concept of «trans depathologization» has jumped to the public agenda, thanks to the emergence of an international movement that advocates for deconstructing entrenched gender stereotypes that are leading this group to be branded as «sick» through a system of overlapping legal, medical and economic issues. In this article I present a general overview of the causes that have led, under the imminent release of new versions of the international manuals of disease DSM-5 and ICD-11, the
\end{abstract}


visibility of these facts, analyzing substantial differences in a set of countries that we will call «from the north» and «from the south», to move on to the analysis of recent events as clues to what may happen in the imminent future in this regard.

Keywords: APA, ICD, DSM, WHO pathologization, trans, queer theory 


\section{Hitos en la internacionalización de la campaña STP2012: de patologización local a interconexión mundial}

La campaña Internacional Stop Trans Pathologization 2012 (a partir de ahora, STP2012) ha conseguido sacar a la palestra un hecho que era poco conocido hace apenas cinco años: las personas trans eran consideradas como enfermas oficialmente, tanto por el estamento médico y sanitario, como por la legalidad, en aquellos países donde esta realidad tenía un reflejo legislativo. En verdad, la consideración de las personas trans como enfermas, si bien ha sido un largo proceso de construcción socio-histórica de identidades abyectas, se produce, oficialmente, en el mismo momento en el cual la orientación homosexual desaparece, para incluir la realidad trans en los manuales internacionales de enfermedades más importantes ${ }^{1}$ : El Manual Diagnóstico y Estadístico de trastornos Mentales (DSM), publicado por la Asociación de Psiquiatría Norteamericana (APA), y el Catálogo Internacional de Enfermedades y otros problemas de salud (CIE) de la Organización Mundial de la Salud (OMS).

La campaña STP2012 tiene sus inicios en el Estado Español, en concreto en Marzo de 2007, día en que se aprueba una ley «de Identidad de Género» que pone el pistoletazo de salida al activismo por la despatologización trans, ya que exige un certificado de trastorno mental y un tratamiento médico, mínimo de dos años, para que las personas puedan acceder a la rectificación registral de nombre y sexo en sus documentos oficiales.

Sin embargo, manifestaciones contra este hecho ya se venían dando a pequeña escala en París, que a finales de los 90 y principios de siglo XXI ya realizaba las manifestaciones «Existrans $»^{2}$ para denunciar la nefasta situación médica y legal a las que estaban sometidas las personas trans de Francia.

Es, por tanto, en 2007 cuando se celebran las primeras manifestaciones por la despatologización trans en el Estado Español en Barcelona y Madrid, además de en París. En el 2008, son 11 ciudades europeas las que organizan

1. Missé Mikel. Y Coll-Planas Gerard. (2010) El Género Desordenado: críticas entorno a la patologizacón de la transexualidad,pag.5, Egales, Barcelona.

2. www.existrans.org consultado el 12-04-2012. 
diferentes manifestaciones. El 2009, es el año en el que varios activistas críticos del Estado Español, que han impulsado las manifestaciones visibilizando la patologización de las personas trans, deciden organizarse y desarrollar formalmente una campaña que toma el nombre de «Stop Trans Pathologization $2012^{3}$. Esta estrategia de planificación y trabajo de redes, potencia la participación de cuarenta y una ciudades de diferentes regiones de todo mundo, en ese año 2009. En Octubre de 2010 (después de la organización del primer Congreso Internacional sobre Derechos Humanos e Identidad de Género, donde activistas trans de todo el mundo establecen alianzas ${ }^{4}$, y donde se celebra la primera manifestación internacional de lucha trans e intersex ${ }^{5}$ ) aumentan a sesenta y una ciudades, y finalmente son setenta, las ciudades de todo el mundo que participan en la edición de 2011, con más de 250 organizaciones y grupos adheridos a la campaña.

Los principales objetivos de la campaña son:

1. La retirada del TIG de los manuales internacionales de diagnóstico (en sus próximas versiones DSM-V y CIE-11),

2. La retirada de la mención de sexo de los documentos oficiales,

3. La abolición de los tratamientos de normalización binaria a personas intersexo,

4. El libre acceso a los tratamientos hormonales y a las cirugías (sin la tutela psiquiátrica),

5. La lucha contra la transfobia: el trabajo para la formación educativa y la inserción social y laboral de las personas trans ${ }^{6}$.

En estos objetivos se vislumbra, por un lado, la perspectiva profundamente provocadora que adquiere el cuestionamiento de los binarios y por ende, el cuestionamiento de las identidades de sexo y género, que hasta el momento consideramos naturales. Y por otra, una realidad geopolíticamente compleja que analizaremos en las siguientes líneas.

3. La fecha del 2012 hacía referencia a la publicación del Manual Diagnóstico y Estadístico de tratornos Mentales (DSM, en su quinta versión), que iba a ser publicado en ese año, aunque finalmente se publicará en Mayo de 2013 (http://www.dsm5.org/Pages/Default. aspx consultado el 01-03-2011).

4. http://www.congenid.org/ consultado el 06-03-2012.

5. http://www.dosmanzanas.com/2010/05/manifestacion-internacional-de-lucha-transintersex-en-barcelona.html consultado el 07-01-2012.

6. http://stp2012.info/old/es/objetivos consultado el 01-05-2012. 


\section{Retos de cara a la despatologización en los Países del «Norte»}

En este apartado trataré de reflejar cuales son los obstáculos que afronta el movimiento por la despatologización trans, en un bloque de países occidentales, de los cuales varios ya poseen leyes «trans» o «Leyes de identidad de Género».

Dejando a un lado el análisis de los países anglófonos no europeos ${ }^{7}$, me dedicaré fundamentalmente, a analizar el contexto europeo con respecto a la cuestión de la despatologización trans.

En este contexto europeo, identifico las siguientes problemáticas y/o retos.

\subsection{Miedo de la población trans a la pérdida de los derechos sanitarios conseguidos}

La exigencia de protección de los derechos sanitarios logrados (aunque fuera a base de declararse enfermos, en muchos casos con el amparo médico-institucional formado por leyes parecidas a la mal llamada «Ley de Identidad de Género $\left.{ }^{8}\right)$ ha sido fuente de desencuentro en el movimiento activista trans. Se argumenta que la efectiva despatologización de las identidades trans, dejaría

7. Dada la relevancia del «lobbying» por la despatologización trans en estos países, especialmente en Estados Unidos, donde se encuentra la Asociación de Psiquiatras Norteamericanos, asociación que edita el catálogo de trastornos mentales DSM (Manual Estadístico de Desórdenes Mentales, catálogo en el que aparece la diversidad trans reducida a su actual término patologizante, la «disforia de género»), que sirve de referencia para el CIE (Catálogo Internacional de Enfermedades, publicado por la Organización Mundial de la Salud, y por tanto catálogo de referencia para los profesionales sanitarios del mundo), pero también para los protocolos sanitarios que se fundamentan en el trastorno mental descritos por la WPATH (Asociación Internacional de Profesionales de la Salud Transgénero, antes conocida como Asociación Internacional de Disforia de Género Harry Benjamín, donde se dictaron los primeros protocolos sanitarios hacia personas trans en el año 1966, y que han variado muy poco, a pesar de los numerosos cambios históricos en torno al hecho trans ocurridos desde entonces).

8. Ley 3/2007, «Reguladora de la rectificación registral de la mención relativa al sexo de las personas» (en http://noticias.juridicas.com/base_datos/Privado/13-2007.html.):asegura únicamente el cambio de la mención de sexo en los documentos oficiales mediante la acreditación de la «disforia de género», sin tener en cuenta, además, los derechos sanitarios ni las actuaciones relativas a la promoción social de un colectivo desfavorecido como es el trans (empleo, formación, educación, etc.). Ausencias que se intentan subsanar con la creación de Leyes Integrales de Transexualidad particulares para cada comunidad española a falta de cambiar una Ley que se queda corta a solo dos años de su aprobación.

Destacar, así mismo, que la primera Ley Integral aprobada, la de la Comunicad de Navarra (en, redactada y promovida por colectivos poco críticos tanto con los partidos en el poder como con las estructuras médico-legales, y que se pretende utilizar como referencia en otros territorios del Estado deja al margen, una vez más, la cuestión de la no exigencia de acreditación de enfermedad para acogerse a ella.

Feminismo/s 19, junio 2012, pp. 143-163 
sin derechos sanitarios tanto en la Sanidad Pública (en aquellos Estados que ahora, con sus más y sus menos, la tienen asegurada), como a nivel de seguros sanitarios privados, en aquellos países donde la sanidad no es pública.

El conflicto está servido: ¿cómo hacer para que el tratamiento transexualizador que incluye tanto el tratamiento hormonal como las cirugías ${ }^{9}$, sea ofrecido por la Seguridad Social o los seguros médicos privados, si no hay un diagnóstico de enfermedad o trastorno de por medio? La brecha abierta por este asunto en la campaña por la despatologización STP2012 es evidente, ya que muchos de los colectivos de los países europeos (como Reino Unido, Dinamarca, etc.), han manifestado su rechazo a esta iniciativa.

Una vez mostrada la postura de estos grupos, cabe preguntarse: ¿estamos dispuestos a defender estos derechos sanitarios? ¿Hasta qué punto? ¿Cómo podemos defender el derecho al acceso sanitario no estando de acuerdo en el discurso que se ha impuesto sobre la concepción de lo que son «los sexos?

Otra pregunta a plantear al respecto: ¿acaso no hay situaciones, no consideradas trastornos ni enfermedades, que reciben atención sanitaria? Efectivamente, podemos encontrarnos con situaciones donde las personas son atendidas en la Sanidad Pública sin que sean catalogadas de trastornadas o enfermedades. Podrían ser ejemplo de esto la atención infantil pediátrica, o de manera más explícita (por hospitalización y uso de quirófano), los partos a consecuencia de embarazos ${ }^{10}$.

Un último argumento a favor de la despatologización es la misma definición del concepto de «salud» que articula la propia OMS antes mencionada: «La Constitución de la OMS define la salud como un estado de completo bienestar físico, mental y social, y no solamente la ausencia de afecciones o enfermedades» ${ }^{11}$.

Aplicando esta definición al caso de la patologización trans, es coherente pensar que el sufrimiento de la población trans nada tiene que ver con trastornos, sino con ese malestar, esa marginación, violencia y exclusión social mencionada en la segunda parte de dicha definición que repercute en la calidad de vida de las personas trans. El verdadero problema se encuentra precisamente ahí, en esa transfobia social fundamentada en el binarismo de la cultura occidental, donde los sexos son clasificados médico-legalmente en

9. Modificación de genitales y cirugías superiores de mamas, tanto de aumento como de eliminación/reducción, pero también cirugías de feminización facial tratamientos y pedagogía foniátricos, tratamientos de eliminación de vello facial y corporal, etc.).

10. que aparecen mencionadas en el manual CIE sin adquirir una dimensión patologizada.

11. Página web oficial de la Organización Mundial de la Salud: http://www.who.int/pehemf/research/agenda/es/index.html consultado el 05-03-2012. 
base a la diferenciación genital ${ }^{12}$, y percibidos socialmente en base a caracteres sexuales primarios y secundarios excluyentes en cada sexo (al contrario que en culturas no occidentales, donde el sexo se construye mediante la interacción de lo biológico y lo cultural, como veremos más adelante), que hace que las personas trans, se sientan disconformes con esas determinadas partes de su anatomía. El mero hecho de la existencia de las identidades trans atestigua que esta dicotomía es una ficción médico-legal, y que por tanto nada tiene que ver con supuestas verdades científicas absolutas.

Es por ello que concluyo diciendo que para conseguir encajar en ese modelo dicotómico occidental de lo masculino/lo femenino y obtener el citado bienestar, es de recibo que las personas trans puedan tener acceso por el sistema público a dichos recursos médicos sin diagnósticos enfermantes de por medio.

\subsection{Justificación biologicista del malestar}

Este segundo escollo, en el camino hacia la despatologización, se refiere al hecho de que muchas personas trans pueden encontrar un alivio al ver que todo el malestar que sienten no es culpa suya, sino atribuible a causas biológicamente determinadas. Dicho de otra forma, el asegurar que estaban predestinadas, por causas congénitas ${ }^{13}$, no solo a sentir una identidad masculina o femenina de la forma en que la sienten, sino a no aceptarse, a odiar su cuerpo, puede ser un gran alivio para muchas personas, ya que no pueden hacer nada al respecto de algo que ya estaba decidido de antemano.

Esta naturalización del sexo ha sido criticada desde distintos ámbitos como la filosofía: «los deseos no son entidades biológicas preexistentes, sino

12. Aunque en los últimos años, y dados los problemas que ha otorgado esta diferenciación simplista se entiende científicamente el sexo como la correlación de varios niveles: sexo genital (pene-vagina) y sexo gonadal (testículos u ovarios) $\rightarrow$ caracteres sexuales primarios, sexo fenotípico (características físicas como pechos, caderas, vello, masa muscular, etc. cuyo desarrollo tiene normalmente un origen hormonal, y por tanto un «sexo hormonal») $\rightarrow$ caracteres sexuales secundarios,.sexo genético (cromosomas XX ó XY). En base a la concordancia de todo ello (aunque frecuentemente se simplifica a la correlación entre sexo genital y gonadal), se identifica al recién nacido con un sexo legal (masculino/femenino).

Sin embargo, ni siquiera estos datos biológicos permiten a veces establecer una frontera clara entre dos únicos sexos (intersexualidad, hecho biológico no obstante patologizado también como «Desorden del Desarrollo Sexual»).

En todo este análisis médico de los sexos se deja de lado la interacción sociocultural del individuo (género), o más allá aún, el sexo/género biográfico (el sentido).

13. Entendidas como aquellos rasgos o entidades presente desde el nacimiento, es decir, adquiridos durante la vida intrauterina. 
«que se constituyen en el curso de prácticas sociales históricamente determinadas», como dice Foucault en su Historia de la sexualidad, o la antropología cultural y la teoría feminista:

(...) que lo biológico sea un prerrequisito previo no significa que, al igual que ocurre con el resto de realidades humanas, se pueda explicar la conducta y la organización social de los individuos de un modo meramente biológico, sin tener en cuenta los importantes condicionantes sociales ${ }^{14}$ (Rubin 1989, 131-132).

Pero también se cuestiona esta concepción esencialista desde ciencias como la sexología, al afirmar desde la sexología sustantiva, mediante la teoría de los estados intersexuales que:

(...) durante milenios se ha creído que un hombre era una persona portadora de un pene. Lo peor es que también se creyó que una mujer era una persona carente de dicho atributo. Una barbaridad ¿verdad?... Todos somos intersexuales puesto que en todos nosotros convive lo masculino y lo femenino... No estamos hablando de monstruos. Lo auténticamente monstruoso es la idea de que el sexo es una regla que debemos cumplir...también es monstruoso desconocer que, con más o menos evidencia y conciencia, todos somos una excepción a esa regla. Y es que esta regla -el sexo- no tiene más que excepciones. Tantas y tal variadas que las llamamos «diversidades $»^{15}$.

La transexualidad no es ni deja de ser una patología; eso es una interpretación moral, subjetiva y humana de algo que estadísticamente es menos abundante. Yo no hablo de patologías, sino de sexuación con sólo dos posibilidades en cada nivel; pero con multitud de posibilidades de mezcla y disposición de esos niveles en cada sujeto. Nada menos y nada más ${ }^{16}$.

Incluso desde la biología, donde, por ejemplo, la bióloga Anne FaustoSterling, desvela que el concepto de identidad sexual está mediada y mediatizada tanto por el ámbito científico como por el sociopolítico ${ }^{17}$.

Ciertamente, podemos argumentar sobre la afirmación anterior: es absurdo determinar que el desprecio de una persona por su cuerpo tiene causas atribuibles a los genes y a procesos bioquímicos que ocurren durante la gestación. Podemos aseverar que la ciencia es la nueva religión de esta era tecnocrática, y como tal suele ser considerada la dadora de verdades. Sin embargo, obviar todos los procesos, referentes e interacciones culturales y biográficos

14. Rubin, Gayle. Reflexionando sobre el sexo: notas para una teoría radical de la sexualidad, en Placer y peligro. Exlorando la sexualidad femenina, coord. Carol Vance, 1989.

15. Landa AJ, Joserra. Sexorum Scientia Vulgata, Revista Española de Sexología, monografía extra-doble $n^{\circ}$ 139-140, Instituto de Sexología, Madrid, 2007.

16. Sáez Sesma, Silverio, Identidad y transexualidad, en Jornadas transexualidad celebradas el 26 y 27 de noviembre de 2004 en Asturias.

17. Fausto-Sterling, A. Cuerpos Sexuados, Melusina, Barcelona,(2006). 
donde se enmarcan la configuración de las identidades masculinas/femeninas a procesos intrauterinos resulta tan reduccionista y fuera de lugar como afirmar que las personas de raza negra poseen una inteligencia inferior a las blancas por el hecho mismo de haber nacido negras (aclarando que se han llegado a realizar estudios científicos que concluían que esto era cierto, sin tener en cuenta la falta de oportunidades).

\subsection{El estamento médico-psiquiátrico como organización de poder}

Las organizaciones médicas, tanto nacionales como internacionales, constituyen actualmente uno de los grupos con más poder en la regulación de ciertas cuestiones de carácter estrictamente sanitario, pero también de cuestiones económicas, tras las que se dejan entrever concepciones ideológicas. En el caso concreto de la variancia de género, nos encontramos con un imbricado conjunto de relaciones entre diferentes asociaciones (como la "World Profesional Association of Transgender Health ${ }^{18}$, que elabora los «Standards of Care», los protocolos sanitarios de referencia (en ningún caso obligatorios, aunque tomados por las organizaciones médicas de las distintas naciones, y por los legisladores como tal en la práctica) destinados a personas trans desde los años 70, que se encuentran en permanente contacto con Kenneth Zucker, Anne Lawrence y Ray Blanchard (principales líderes del grupo de estudio del «trastorno de identidad de Género» de la Asociación de Psiquiatría Norteamericana. ${ }^{19}$ ). Por tanto, en el caso del tratamiento sanitario de la alternancia de género, vemos que distintas asociaciones coinciden y parten de la premisa de los criterios patologizadores de una asociación de psiquiatras de un solo país, que necesitan de dichos criterios separadores de lo «normal/anormal» para mantener el orden occidental tradicional (que no se cumple en otras muchas sociedades, como veremos) de los sexos. Así, el discurso biomédico sobre la diferenciación sexual, basado en un modelo simplista, se torna en un discurso que se convierte en dogma de base y que es difícil de que llegue a ser cuestionado.

Hay que señalar, además, que el poner en duda las claves por las cuales se produce la patologización de la variabilidad de género, no solo cuestiona el

18. http://www.wpath.org/ (antes conocida como Harry Benjamin International Gender Dysphoria Association, HBIGDA) consultado el 15-10-2011.

19. Zucker, Kenneth J. and Lawrence, Anne A.(2009)'Epidemiology of Gender Identity Disorder: Recommendations for the Standards of Care of the World Professional Association for Transgender Health',International Journal of Transgenderism,11:1,8-18.

(artículo donde Zucker y Lawrence dan recomendaciones sobre los «Standards of Care» a los profesionales de la WPATH) 
poder otorgado al estamento médico-psiquiátrico como dotador de»la verdad incontestable», sino que además pone en juego algo mucho más material: los beneficios económicos que adquieren mediante la aplicación rigurosa, explícita y extensiva de estos criterios y protocolos, de los cuales estos profesionales especializados en la problemática trans son el único referente para la población general, que no tiene una noción propia de las cuestiones de sexo/ género, para las personas trans, como para los organismos nacionales encargados de regular protocolos en materia de derechos sanitarios y legales. Así, asistimos a un pingüe reparto de beneficios entre varios psiquiatras y psicólogos clínicos, cirujanos, endocrinos etc. especializados en «lo trans», que se encuentran sin competencia directa reconocida.

Por tanto, concluyo diciendo que es conocido el poder que los médicos tienen sobre sus pacientes y sus familias, pero quizás es menos conocido de qué modo ese mismo poder médico hegemónico es servil a una ideología cultural intransigente y patriarcal, y además capitalista. Así, aquellas personas que no estén en alto grado de conflicto con su cuerpo (a pesar de los múltiples esfuerzos ajenos descritos), si lo único que desean es hacer un cambio legal en su documentación atendiendo a su sentir psicosocial, no encajarán en este modelo biomédico del trastorno "por malestar», ya que estarán cuestionando los paradigmas que rigen tanto los protocolos sanitarios (la medicalización obligatoria), como la legislación: el binarismo, la jerarquía patriarcal, y el beneficio económico. Si estas personas no mienten en sus evaluaciones psiquiátricas y no se someten a dichos protocolos médicos, quedarán excluidas de su derecho a verse reconocidas en el género que sienten.

\subsection{Legalidad trans distinta en cada país}

El hecho de que estos países consideren la necesidad de comprobar mediante documentos de identificación pública (DNI, pasaporte etc.) el sexo al que pertenece una persona, es, en muchos casos, uno de los pocos factores que tienen en común las legislaciones trans. Así, todos los estados consideran fundamental controlar el sexo de las personas a nivel legal, para que queden claramente identificadas como hombres o como mujeres ${ }^{20}$ (por mucho que este

20. Recordemos el caso reciente de la India, donde los»hijra» (colectivo compuesto principalmente de personas trans e intersexos) han podido cambiar su sexo legal a un tercer sexo (Hombre/Mujer/Otro). Dejando de lado las críticas que ha recibido la medida (por un lado, evita problemas a las personas en situaciones donde deben presentar sus documentación oficial cuando su apariencia no se ajusta a su sexo legal inicial. En contrapartida las deja expuestas a la violencia tránsfoba del resto del colectivo, al quedar claramente identificadas como «no hombre» $\mathrm{y}$ «no mujer»). Ni siquiera una media 
criterio falle constantemente, no solo en el caso de muchas personas trans, sino también en caso de población no-trans que no se ajusta a la apariencia culturalmente asociada tanto a lo masculino como a lo femenino).Una vez más, se ejerce un control sobre el sexo de los individuos sin tener en cuenta las variabilidades de género que puedan darse, reflejo, una vez más, de la visión restrictiva que tiene occidente de los sexos, y valorándolo como característica que identifica inconfundiblemente a sus ciudadanos (cuando también podrían aparecer en los documentos otras características como la estatura o la raza/etnia del sujeto, factores que suelen permanecer invariables a lo largo de la vida de las personas, pero que en el mundo que habitamos, y después de la lucha por la liberación que han llevado a cabo durante años las personas negras, sería «políticamente incorrecto» incluirlas en los documentos públicos).

Una de las exigencias de la campaña STP2012 que mayores fricciones ha provocado en Europa ha sido precisamente la petición de que la mención de sexo desaparezca (tanto para las personas trans como par las que no), por ser el sexo una cualidad que pertenece al ámbito privado, y por ser incoherente tener que mostrarlo públicamente (y por tanto, ser proclive a discriminaciones en países con culturas históricamente patriarcales) con el objetivo de igualdad entre sexos que se persigue en los estados occidentales ${ }^{21}$. La admisión de esta exigencia, en línea con el objetivo general de la despatologización de las identidades trans, abre la posibilidad de que la legalidad haga efectiva una verdadera plasmación de la diversidad de sexos y géneros, y por tanto sería la prueba patente de que el sistema catalogador de sexos actual es irreal e inservible. Los gobiernos de dichos Estados, como garantes de un orden social fundamentado en el «dogma» irrebatible de la verdad absoluta de los sexos, no pueden asumir a día de hoy esta demanda.

El rechazo a plantear esta medida ha venido mayoritariamente de mano de algunos activistas trans, ya que después de largo tiempo de sufrimiento ven en el cambio de la mención de sexo en los citados documentos un logro, un espaldarazo a que el camino que han tomado es el correcto, y que pude ser utilizado como defensa para quien cuestione el sexo de esa persona

similar (que sigue jerarquizando las categorías de sexo), podría ser posible en un país occidental, por su rígida ideología de los sexos.

21. Debemos distinguir entre los documentos públicos que suelen ser mostrados en los trámites y transacciones que realizamos en el día a día, de los registros civiles y documentos administrativos, que sí pueden contener esta información clasificada, pero que permanecen en el ámbito privado, a fin de poder conseguir estadísticas y aplicar herramientas y políticas de igualdad. 
(cuestionamiento y reacción que se producen, una vez más, en el marco de una concepción rígida del sexo que la misma vida se empeña en desbaratar).

Más allá del mero hecho de que siga vigente la mención de sexo en los documentos oficiales, nos enfrentamos a una situación donde los procedimientos para conseguir el cambio legal son muy distintos de un país a otro (en el caso de que esta posibilidad exista, aunque en todas ellas se refleja la dura sanción que espera a las personas trans si inician cualquier acción destinada a cambiar este sexo legal.

Así, hallamos legislaciones mucho más sancionadoras como la legislación de cambio de sexo alemana, que obliga a tener que pasar por un juicio donde se evalúa al sujeto (en vez de ir al registro e iniciar un sencillo trámite administrativo) y donde habrá que acreditar: la esterilidad de la persona (violando así sus derechos reproductivos ${ }^{22}$ ), el haber pasado por cirugías obligatorias (aumento de pechos o mastectomía y cirugías de reasignación sexual ${ }^{23}$ ), tener que ser solterx o si no divorciarse obligatoriamente. Se exigen, además, los requisitos médicos establecidos como referente, (no como obligación, aunque parece que los médicos y los juristas se escuden, cada vez más, en que son protocolos de obligado cumplimento) de estándares de salud trans internacional por la antes mencionada WPATH: el certificado de estar afectadx de «disforia de género» junto con una hormonación obligatoria mínima de dos años (cuyo resultado es la mencionada esterilización química de la persona en la mayor parte de los casos), acompañado frecuentemente de un control semanal del comportamiento masculino o femenino del individuo, según el caso, del individuo por parte de psiquiatra o psicólogo clínico.

Frente al caso ejemplar de Alemania nos encontramos con una percepción a nivel legal de lo que significa ser hombre/mujer distinta, mucho más laxa en comparación con las leyes alemanas, pero absolutamente sancionadora (una vez más ${ }^{24}$ ), y controladora del normal discurrir entre la expresión de género

22. Los derechos sexuales y reproductivos se pueden resumir en que cualquier persona tiene derecho a decidir con quién, cuando y cómo tiene, o no hijos y relaciones sexuales. Son los derechos que garantizan la libre decisión sobre la manera de vivir el propio cuerpo en las esferas sexual y reproductiva.

23. Obsérvese que la utilización extensa del concepto «cirugías de reasignación sexual»en vez de «cirugías de modificación genital» denota la noción de que el sentir ser hombre o mujer se encuentra única y exclusivamente en nuestra genitalidad. Así, podríamos llegar a la conclusión de que una persona que es biológica y psicológicamente hombre, y que sufra una amputación de su pene, dejaría de serlo..para pasar a ser mujer.

24. Podemos afirmar que no hay ninguna ley ningún país de Europa que no sancione al individuo que quiera cambiar su mención de sexo y/o su nombre. Para comprobarlo, podemos acudir a los resúmenes con los requisitos de cada país al respecto del estado civil y del nombre publicados en la web de la organización TGEU (Transgender Europe): 
de la persona como sería el caso de la «Ley de Identidad de Género» Española. Su aporte más significativo es que, a parte reconvertir el juicio en un trámite administrativo, admite la existencia de mujeres con pene y hombres con vagina (ya que menciona que el sexo de las personas es psicosocial y no depende de la biología, y retira, por tanto, la obligatoriedad de las cirugías genitales), incurriendo en una absoluta contradicción a esa descripción psicosocial del sexo y dando legitimidad legal a la noción biológica de sexo al incluir en la Ley el requisito de acreditar «Disforia de Género», junto con la obligación de modificar las características morfológicas del cuerpo con un tratamiento hormonal de al menos dos años y la también antes mencionada evaluación frecuente del profesional de salud mental. Además, España incurre en una mayor restricción en el cambio de nombre, ya que a diferencia de otros países europeos (como Bélgica o recientemente Suiza, donde de un día para otro se puede modificar el nombre hacia el sentido como propio), establece en su Ley 40/99, de 5 de noviembre sobre nombre y apellidos que «quedan prohibidos los nombres que induzcan en su conjunto a error en cuanto al sexo ${ }^{25}$.

Como observamos, las leyes que expresan cuales son los requisitos para pertenecer a uno u otro sexo son bastante diferentes, pero persiste en todas el ánimo de mantener y preservar una de las bases sobre la que se sustentan las sociedades occidentales, la distinción única y exclusiva de una forma de ser hombre y otra de ser mujer.

Es por ello que debemos de entender y adaptar nuestras estrategias de cara a estas realidades, ya que las personas (legisladores y profesionales sanitarios, y la población en general, sean trans o no), están mediatizadas por la concepción que se tiene de lo que es el sexo y el género en cada territorio, y

http://tgeu.net/PubAr/Documents/Co01/Compilation_CivilStatus.pdf consultado el 28-03-2012 y http://tgeu.net/PubAr/Documents/Co01/Compilation_RightOfName.pdf (recursos en inglés) consultado el 28-03-2012.

25. Dándose, en el caso español, la perversidad, mediante la interacción de la «Ley de nombres y apellidos» y de la «Ley de Identidad de Género», de que si una persona quiere pasar, por ejemplo, de llamarse Juan a llamarse Pedro lo pueda realizar de un día para otro, y sin embargo si esa misma persona quiere llamarse Sara, tenga que soportar un certificado de trastorno mental, y dos años de tratamiento médico y control por parte del psiquiatra o psicólogo clínico, obligando en muchos casos, a permanecer este periodo en un absoluto ostracismo social y laboral.

Por lo demás, una persona que desee modificar su mención de sexo pero no su nombre (por resultarle cómodo, haberse socializado con él, etc), no podrá tener derecho a su propio nombre si en el registro ese nombre queda identificado como «masculino», pues en el registro constan completos listados de nombres «de hombre», listados de nombres «de mujer», y un reducido grupo de nombres que se han utilizan culturalmente para ambos sexos. 
por tanto es una barrera en cuanto a prioridades, donde la reivindicación de la despatologización queda totalmente oculta.

\section{Retos de cara a la despatologización en los Países del «Sur»}

En el panorama de los países en vías de desarrollo nos encontramos con una situación distinta, menos estructurada y jerarquizada tanto a nivel sanitario como a nivel legal, y fundamentalmente en cuanto a su cultura sexual y de género se refiere, situación a priori más abierta a oportunidades de cambio pero que con la globalización de la economía y la cultura occidentales de por medio, corre el riesgo de homogeneizarse y de ser patologizada:

\subsection{Culturas distintas en la concepción de sexo/género en poblaciones aisladas vs. Violencia institucionalizada y socializada en los núcleos urbanos}

Nos encontramos con un contexto donde hay culturas en las cuales se ha dado una tradición transgenerista ${ }^{26}$. Así, en las culturas donde existe un tercer género (Ejemplos varios: En la cultura navajo existen tres géneros: varones, mujeres y nadle), la asignación de un individuo como nadle suele determinarse si exhiben rasgos genitales ambiguos. Existen también «falsos» nadle, cuyos rasgos genitales son definidos, pero que se comportan y son reconocidos como nadles «auténticos». También están las tradiciones berdache (dos-espíritus: masculino y femenino en un solo cuerpo) de culturas nativas norteamericanas o el tercer género mahu polinesio. En distintas culturas africanas existen además tradiciones de matrimonio entre mujeres y matrimonio entre muchachos que, en ocasiones, disuelven las diferencias entre categorías, como los matrimonios entre guerreros solteros y muchachos jóvenes, donde al muchacho joven que contraía matrimonio con el guerrero se le consideraba una mujer y las prácticas sexuales de ambos eran consideradas lícitas y no categorizadas como homosexuales. Por último tenemos también en el Pacífico los rituales de Género cruzados, como en sociedades de Papúa Nueva Guinea ${ }^{27}$ Pero también en América latina nos encontramos tradiciones transgeneristas como las machas y los hembros mestizos de la costa del

26. Denominamos transgeneristas a aquellas personas que transitan en su rol social sin necesidad de intervenir su cuerpo, sino haciendo uso solo de tecnologías estéticas exterior (ropa, maquillajes /pintura corporal, cabellos, etc). Frente a ella encontramos el término «transexual», palabra procedente de la medicina que designa a aquellos sujetos que sienten la imperiosa necesidad de modificar su estructura corporal mediante componente químicos(hormonas) y/o cirugías.

27. Nieto Piñeroba, J.A (2004)., Globalización y transgenerismo en el área del Pacífico, Revista Española del Pacífico, n. 16. 
Ecuador, que no esconden sus características sexuales secundarias no intervenidas ${ }^{28}$, o la cultura huaoran del amazonas, donde la sensualidad no se centra en los genitales ni es dominio exclusivo de la heterosexualidad adulta. No sexualizan la sensualidad y los distintos placeres corporales permanecen indistinguidos e identificados como bienestar en su vida común. No existe para esta cultura una noción semejante a nuestro concepto de sexualidad, ni categorizaciones que distingan unos comportamientos sexuales de otros, incluso para aquellos en que participan niños, jóvenes y adultos. Tampoco es significativa, en su construcción de los géneros, la atracción sexual entre hombres y mujeres.

Estas poblaciones transgeneristas (incluyendo identidades más normativas, como las kathoey tailandesas, en la concepción de sexo/género propia de occidente), a parte de una concepción distinta sobre el sexo, el género y la sexualidad (presentes incluso en un lenguaje poco generizado con respecto a las lenguas de Occidente), tienen algunas notables diferencias con respecto a la población trans occidental: el concepto de transición social o bien no existe, o se realiza muy temprano en la vida, y a la vez se les otorgan papeles relevantes en estas colectividades (como médiums o sanadorxs de espíritus $)^{29}$.

En todas estas culturas, las clasificaciones hechas por el modelo biomédico quedan anuladas, y tanto las cirugías de «resignación» (que en muchos casos no se plantean o se desconocen) como las categorías legales institucionales de masculino/femenino (o varón/hembra según la nomenclatura legal de cada país), pierden su relevancia, y la supuestamente validez absoluta de dichos postulados binarios queda en entredicho. Sin embargo, observamos que el sistema binario de sexo/género occidental se implanta con cada vez más fuerza en estos territorios, con la globalización cultural y económica de por medio.

Frente a estas poblaciones, que permanecen o han permanecido aisladas al estilo de vida y la ideología de géneros marcado por Occidente, nos encontramos con la situación dual de que en muchos de estos países convive esta realidad de plasticidad de los géneros con un panorama en el que no solo

28. Elizabeth Vásquez del Proyecto Transgénero Ecuador (http://www.proyecto-transgenero.org/ consultado el 20-03-2011) en la conferencia «Reflexiones no binaristas sobre el feminismo», Jornada Feministas Estatales en Granada, 2009 (vídeo en: http://transgranadafeminista.blogspot.com/).

29. Winter, Sam(2009)'Cultural Considerations for the World Professional Association for Transgender Health's Standards of Care: The Asian Perspective', International Journal of Transgenderism,11:1,19-41. 
hay falta de legislación anti-discriminación, sino directamente una situación insoportable de homofobia y transfobia institucionalizada, que se concreta en leyes con fuertes castigos (incluso la muerte), acompañada de una violencia exacerbada hacia las personas variantes de género que son habitualmente rechazadas socialmente. Comunes son los asesinatos y las agresiones hacia la población trans en núcleos urbanos, bastante más elevados que los existentes en los países «del Norte» ${ }^{30}$.

Observamos que la violencia con producto de muerte es especialmente alta en los países latinoamericanos. El hecho de que en estos países confluya la cultura postcolonial occidental con una situación de subdesarrollo económico, ha transformado las sociedades locales en altamente peligrosas para las personas trans, que cuestionan ambos polos (el binomio de sexo/género cultural, así como el estilo de vida «alternativo» en términos económicos).

Una de las mayores críticas que se ha hecho a la campaña STP2012 es que tiene una posición primermundista y occidental. Sin embargo, observamos, por ejemplo, que en la web de la campaña ${ }^{31}$, queda patente la diferencia entre los países del primer mundo, en contraste con el apoyo masivo de aquellos países en vías de desarrollo (especialmente los países latinoamericanos), donde, a pesar de que en muchos de ellos no existe una legislación patologizante ni hay una atención sanitaria protocolizada especialmente para estas personas, los efectos de la violencia que viven estos colectivos han hecho que se vuelquen en esta campaña que, a priori, nada tiene que con la situación en la que viven su día a día.

Así, en estos núcleos urbanos, donde la cultura de derechos humanos no está tan desarrollada como en los países del «Norte», la situación es similar a la vivida en los países europeos a finales de los 70 y principios de los 80 , donde la fobia estaba institucionalizada y socializada ${ }^{32}$. Lxs activistas trans de entonces vieron como un logro que de una concepción de ellxs como criminales se pasara a una situación donde se les dotaba de cierta entidad y derechos que antes no poseían, aunque fuera en calidad de «enfermxs».

30. Observatorio de personas trans asesinadas, en http://www.tgeu.org/tdor2009 espa\%C3\%B1ol consultado el 01-12-2011.

31. en la sección de adhesiones de http://stp2012.wordpress.com/support/ el 01-10-2011)

32. Ley de Vagos y Maleantes (1922) reformada en 1954, a la que le sucedió la Ley de Peligrosidad y Rehabilitación Social (LPRS) de 1970.

Platero, Raquel (2009). Discriminación por orientación sexual e identidad de género. En Álvarez, Enrique, Figueruelo, Ángela y Nuño Laura (dir). Estudios interdisciplinares sobre igualdad. Madrid: Iustel. 169-182. 
Por tanto, el proceso de negociación y protesta colectiva que pueda darse en estos lugares puede culminar, en el futuro, en una situación donde se acabe recurriendo, una vez más, a la patología biomédica globalizada por occidente como moneda de cambio.

\subsection{Sanidad trans ausente y mercados económicos de salud y estética trans sumergidos}

Al igual que las leyes de «Identidad de Género», inexistentes en muchos de estos países y sustituidas por un clima de ostracismo social y legal, tampoco encontramos, lógicamente, una atención sanitaria que recoja y solvente las necesidades de salud de las personas trans. ¿Hasta qué punto un cambio en los manuales internacionales de enfermedades incidiría en países donde no hay sanidad pública a la que acceder?

Este hecho, tanto una situación de debilidad estructural de las personas trans de estos países, como una oportunidad de cara a no cometer el error de caer en la patologización, se ve afectado por el hecho de la globalización cultural antes mencionada.

Así, ante las necesidades de modificación corporal de una sociedad binaria, pobre y por ende altamente tránsfoba, magnificadas por la necesidad de salir adelante (y por tanto de modificar el cuerpo en base a los estrictos patrones de representación de la masculinidad y la feminidad, incluso para poder trabajar, como en el caso de las prostitutas trans) hace que hayan surgido oportunidades de negocio en el mercado sumergido de la modificación corporal y la estética trans. Esto mercados sumergidos afectan, como en toda situación estructural de violencia patriarcal, en una mayor proporción a las mujeres trans.

Podemos mencionar, por ejemplo, la compra y posterior autoinyección de silicona liquida, comprada por estas mujeres en maneras absolutamente alegales y sin ninguna garantía para la salud en el mercado negro, o el tráfico de hormonas sin ningún control, que acaban causando incluso las muertes de las usuarias.

Además, pseudo-profesionales de la salud ofertan cirugías de modificación corporal sin ningún tipo de control, y cambiando constantemente el precio de sus servicios, ante la falta de regulación existente. 
4. A modo de conclusión: avances recientes y empeños patologizadores. ¿Un cambio de paradigma?

En tiempos recientes, a raíz de la entrada de la campaña stp2012 y del concepto «patologización trans» en la agenda pública, estamos asistiendo a una miríada de declaraciones y gestos de instituciones nacionales e internacionales de gesto distinto en cuanto a despatologización trans se refiere.

Por un lado, tenemos declaraciones estatales e internacionales a favor de la despsiquiatrización: del comisario de Derechos Humanos del Consejo de Europa $^{33}$, el gobierno español (entre otros estados) ${ }^{34}$, asociaciones internacionales de profesionales como WPATH ${ }^{35}$ y SOCUMES ${ }^{36}$ y por supuesto las redes de organizaciones activistas trans internacionales (TGEU, ILGA Europe, MSM-GF, Transgender Network Asia)

Sin embargo, a la vez asistimos a la continuista voluntad de clasificación de las identidades trans en el DSM-5 ya expresada en diferentes borradores publicados por la Sociedad Americana de Psiquiatría ${ }^{37}$, y a un persistente modelo actual de atención sanitaria a personas trans basado en la evaluación y autorización externa.

En los últimos días han ocurrido hitos importantes que sitúan a la despatologización como un hecho alcanzable:

La aprobación y promulgación de la ley «de Identidad de Género» Argentina el 24 de Mayo de $2012^{38}$, primera ley en el mundo en el que no se exige ningún diagnóstico determinado para poder acceder a un cambio de documentación y que en base al consentimiento informado de la propia persona interesada permite el acceso a tratamientos y cirugías de transición. Esta ley se convertirá en un símbolo y un referente en cuanto a que sí se puede conseguir una despatologización total, tanto legal como en el acceso a sanidad trans-específica.

La publicación, el 17 de Mayo de 2012 de un informe por parte de un grupo de trabajo de expertxs (varixs de ellxs estrechamente ligados a la campaña STP2012) para analizar cómo garantizar la permanencia de la realidad

33. http://www.transrespect-transphobia.org/uploads/downloads/Publications/Hberg es.pdf, consultado el 01-05-2012.

34. http://www.dosmanzanas.com/2010/05/el-gobierno-espanol-apuesta-formalmentepor-la-despatologizacion-de-la-transexualidad.html consultado el 01-05-2012.

35. http://www.cpath.ca/2010/06/13/de-psychopathologisation/ consultado el 01-05-2012.

36. http://maspsicologia.com/2010/02/declaracion-de-la-socumes-sobre-despatologizacion-de-la-transexualidad/ consultado el 01-05-2012.

37. http://www.psych.org/ consultado el 17-05-2012.

38. http://www.boletinoficial.gov.ar consultado el 24-05-2012. 
trans, y por tanto del acceso sanitario en un manual usado en todo el mundo como es el Catálogo Internacional de Enfermedades y otros problemas de salud de la OMS, despatologizando a la vez esta mención en el manual (en la parte final del catálogo sobre «situaciones que proclives a ser tratadas en los servicios de salud» sin que se las considere enfermedades. Opción que en el manual DSM de los psiquiatras norteamericanos, debido a su estructura en la que clasifica únicamente estados patológicos mentales, no es posible).. Lo característico del grupo de trabajo, reunido en La Haya (Países Bajos), con parte del equipo de gobierno holandés, es que estuvo asesorado por 2 importantes figuras de la OMS: el coordinador de la revisión del capítulo de trastornos mentales del nuevo Catálogo Internacional de Enfermedades de la OMS (versión 11, que se publicará finalmente en 2015) y la coordinadora del grupo de trabajo sobre salud sexual. Todo augura a que autoridades de la OMS escuchen las pautas indicadas por este grupo de expertos, con lo cual si la fórmula de despatologizar manteniendo a la vez «lo trans» en el CIE se lleva a cabo, el DSM-5, que se publica finalmente en 2013, se vería obligado a modificar sus criterios, al haber una disparidad de clasificación en la inclusión del trastorno con respecto al capçitulo equivalente del CIE- $11^{39}$.

Veremos, por tanto, si la campaña STP2012 y el activismo por la despatologización trans alcanzan un punto de no retorno en 2015. Allí nos encontraremos.

\section{Referencias bibliografícas}

Fausto-Sterling, Anne. Cuerpos Sexuados, Melusina, Barcelona, 2006.

FOUCAUlt, Michel. Historia de la sexualidad, 3 vol. España Siglo XXI, 1987.

LANDA, Joserra. Sexorum Scientia Vulgata Revista Española de Sexología, monografía extra-doble $n^{\circ}$ 139-140, Instituto de Sexología, Madrid, 2007

LEY 5/2007. Reguladora de la rectificación registral de la mención relativa al sexo de las personas» (en http://noticias.juridicas.com/base_datos/Privado/l32007.html.)

MissÉ Mikel. Y COLL-PlANAS Gerard. El Género Desordenado: críticas entorno a la patologizacón de la transexualidad, Egales, Barcelona, 2010

Nieto PiÑeroba, José Antonio. «Globalización y transgenerismo en el área del Pacífico», Revista Española del Pacífico, n.16, pp. 22-25, 2004.

39. Informe descargable en http://transactivists.org/2012/05/20/es-tiempo-de-reformacuestiones-de-salud-trans-en-la-clasificacion-internacional-de-enfermedades/ consultado el 20-05-2012. 
RUBIN, Gayle. «Reflexionando sobre el sexo: notas para una teoría radical de la sexualidad, en VANCE, Carole (Comp). Placer y peligro. Exlorando la sexualidad femenina. Talasa, Madrid. 1989, pp.113-190

SAÉz SESMA, Silverio. Identidad y transexualidad, en Transexualidad, Consejo de la Juventud de Asturias, 2004.

Winter, Sam. (2009)»Cultural Considerations for the World Professional Association for Transgender Health's Standards of Care: The Asian Perspective', International Journal of Transgenderism, Volumen 11,2009, pp.19-41

ZuCKer, Kenneth J. \& LAWRENCE Anne A. «Epidemiology of Gender Identity Disorder: Recommendations for the Standards of Care of the World Professional Association for Transgender Health». International Journal of Transgenderism. Volumen 11, 2009, pp. 8-18.

\section{Web consultadas}

Boletin Oficial de la República de Argentina: http://www.boletinoficial.gov.ar

Página web oficial de la Organización Mundial de la Salud: http://www.who.int/ peh-emf/research/agenda/es/index.html

Web campaña STP2012: http://www.stp2012.info/old/

Web Congreso Internacional de Derechos Humanos e Identidad de Género http:// www.congenid.org/

Web proyecto Transrespect vs. Transphobia: http://www.transrespect-transphobia.org/uploads/downloads/Publications/Hberg_es.pdf,

Web Asociación de Psiquiatría Norteamericana: http://www.psych.org/

Web del Manual DSM-5: http://www.dsm5.org/Pages/Default.aspx

Web Derechos humanos http://www.mujeresenred.net/IMG/pdf/Milu_Vargas.pdf

Web Asociación Profesional Internacional sobre la Salud Transgénero: http:// www.wpath.org/

Web del proyecto transgénero: http://www.proyecto-transgenero.org/

Web noticias LGTB Dosmanzanas: http://www.dosmanzanas.com/2010/05/elgobierno-espanol-apuesta-formalmente-por-la-despatologizacion-de-la-transexualidad.html

Web Asociación Canadiense de Profesionales de la Salud Trans: http://www.cpath. ca/2010/06/13/de-psychopathologisation/

Web SOCUMES: http://maspsicologia.com/2010/02/ declaracion-de-la-socumes-sobre-despatologizacion-de-la-transexualidad/

Web de las jornadas estatales feministas de Granada: Juana Ramos: En busca de la esencia perdida: las identidades desde una perspectiva trans, http://www.feministas.org/IMG/pdf/Juana_Ramos.pdf. 
Diversidades cuestionadoras: la campaña internacional «stop trans patologización... 163

Blog consultados

Blog de la campaña «Stop Trans Pathologization 2012»: http://stp2012.wordpress.com/support/),

Blog de las manifestaciones Existrans de Paris: www.existrans.org

Blog sobre las Jornadas Feministas Estatales de Granada 2009: http://transgranadafeminista.blogspot.com

Blog de la Acera del Frente: http://aceradelfrente.blogspot.com/ 